\title{
Avaliação da Presencialidade em um Fórum LV utilizando Lógica Fuzzy
}

\author{
Monck Charles N. de Albuqueruqe ${ }^{1}$, Gilvandenys Leite Sales ${ }^{1}$, \\ Pedro Pedrosa Rebouças Filho ${ }^{1}$, Cláudio Marques de Sá Medeiros ${ }^{1}$ \\ ${ }^{1}$ Programa de Pós-Graduação em Ciência da Computação - PPGCC \\ Instituto Federal de Educação, Ciência e Tecnologia do Ceará - IFCE \\ Av. Treze de Maio, 2081 - Benfica - 60.040-215 - Fortaleza - CE - Brazil \\ \{monckcna, denyssales, pedrosarf, claudiosa1965\}@gmail.com
}

\begin{abstract}
This paper presents a proposal of the evaluation of Social Presence in virtual environments. Fuzzy Logic was applied in LMS Moodle forums that use the LV Model Evaluation. The LV is a process evaluation that makes use of qualitative mentions associated to an iconographic language. After the process of fuzzification and defuzzification, we have in the output a quantitative value that corresponds to the degree of presence of the student in the forum. The research was applied in a specialization course of the Federal University of Ceará (UFC). As a result, the computational design to have a model capable of measuring the student's presence with prospects of being applied to other evaluation tools present in LMS was obtained.
\end{abstract}

Resumo. Este artigo apresenta uma proposta de avaliação da Presença Social em ambientes virtuais. Foi aplicada Lógica Fuzzy em fóruns do AVA Moodle que utilizam o Modelo LV de avaliação. O LV é uma avaliação processual que faz uso de menções qualitativas associadas a uma linguagem iconográfica. Após o processo de fuzzificação e defuzzificação, tem-se na saída um valor quantitativo que corresponde ao grau de presencialidade do aluno no fórum. A pesquisa foi aplicada em um curso de especialização da Universidade Federal do Ceará (UFC). Obteve-se como resultado a concepção computacional de um modelo capaz de mensurar a presencialidade do aluno com perspectivas de ser aplicada a outras ferramentas de avaliação presentes nos AVAs.

\section{Introdução}

O avanço da Internet, das redes sociais e suas ferramentas fez nascer um novo comportamento: o de sentimento de pertencimento a um grupo. Neste contexto é relevante a presença virtual, a qual se identifica com a capacidade que cada pessoa tem de interagir utilizando diversas tecnologias e em diversos contextos para se comunicarem com outras pessoas geograficamente distantes. Avaliar esse comportamento tem sido um desafio dentre aqueles que se valem de Tecnologias Digitais da Informação e Comunicação (TDIC) como suporte à aprendizagem.

Pesquisadores como Kim et al. (2016), Bizzo (2009), Silva (2012) e Valente (2000) vem estudando a presencialidade dos alunos em ambientes virtuais de aprendizagem e indicam que essa presencialidade é importante para o processo de ensino e aprendizagem. 
VI Congresso Brasileiro de Informática na Educação (CBIE 2017)

Anais do XXVIII Simpósio Brasileiro de Informática na Educação (SBIE 2017)

Na modalidade de Educação a Distância $(\mathrm{EaD})$, o fator presencialidade vem ganhando destaque, e a aferição da presencialidade em Ambientes Virtuais de Aprendizagem (AVA) pode ser significativa para o processo de avaliação da aprendizagem. Nestes ambientes virtuais, diversas atividades síncronas e assíncronas propiciam o engajamento do aluno na resolução de problemas e na sua socialização. A avaliação de sua participação e interação podem caracterizar a presencialidade no AVA, o que demarca sua Presença Social (PS).

Segundo Tu (2002) apud (Basto 2011), PS é o grau de sentimento da percepção de pertencimento no grupo, na comunidade em interação, e reação ao conectar-se com outros indivíduos por recursos de comunicação mediada por computador.

Silva (2012) afirma que "é importante para o professor conhecer os graus de PS de seus alunos no decorrer de um curso, de modo que possa, se necessário, intervir de algum modo", o que implica avaliar.

Nos últimos 10 anos, pesquisadores como Bai and Chen (2008), Saleh and Kim (2009) e Arias et al. (2012) têm utilizado a teoria dos conjuntos difusos para realizar avaliação de desempenho de alunos. Foram propostos diversos modelos com a inclusão da avaliação na modalidade presencial e à distância.

Bai and Chen (2008) propuseram um método que usa funções de pertinência e regras difusas para determinar o desempenho de alunos através de resolução de questões. Para encontrar o grau de dificuldade foram analisados os acertos das respostas dos alunos e do tempo gasto para responder às questões. A complexidade e a importância são fatores de ponderação determinados pelo especialista no domínio. No entanto, o método de Bai and Chen (2008) buscava avaliar apenas o desempenho do aluno em uma tarefa.

No trabalho de Saleh and Kim (2009) foi proposto um sistema de avaliação difusa que melhora o método de Bai and Chen (2008). Consideraram também os três fatores mencionados por outros autores como Weon and Kim (2001): a dificuldade, a complexidade e importância das questões. Para determinar o desempenho de alunos, Saleh and Kim (2009) utilizaram um sistema composto por três controladores difusos (Fuzzy Logic Controller - FLC). Os controladores estão interligados sequencialmente, em que a saída do último FLC é o valor requerido para calcular a pontuação final dos alunos de uma forma mais precisa, mediante o uso do acerto das respostas.

$\mathrm{Na}$ pesquisa de Arias et al. (2012), o autor propôs a multidimensionalidade da avaliação de desempenho como um resultado de interações complexas, empregando uma metodologia de avaliação que faz uso da lógica difusa, é capaz de aferir o aprendizado dos estudantes. Sendo utilizado o software educacional Conexão do Saber aplicada no quinto ano do ensino fundamental na disciplina de matemática.

Diante das altas taxas de desistência em cursos EaD, Silva (2012) investigou a correlação entre afetividade e a emoção nos fóruns e chats com a evasão em curso de EaD. Foi criado um mecanismo automático para analisar a PS através da mineração de dados utilizando os software WordSmith e Eurekha. No entanto, há a necessidade de cadastrar um grande vocabulário de termos e expressões para o seu funcionamento.

Seguindo a linha das pesquisas dos autores citados, este trabalho está fundamentado na teoria dos conjuntos difusos [Zadeh 1965] e no Modelo LV de avaliação formativa 
VI Congresso Brasileiro de Informática na Educação (CBIE 2017)

Anais do XXVIII Simpósio Brasileiro de Informática na Educação (SBIE 2017)

e seus Vetores-Aprendizagem [Sales 2010]. Optou-se em utilizar o fórum de discussão simples do AVA Moodle por ser uma ferramenta assíncrona, em que o AVA registra todos os logs de interações dos alunos na plataforma. A partir desses dados, cria-se uma base dessas interações e através da utilização da Lógica Fuzzy é possível analisar essas interações e extrair um fator de presencialidade (FP) que servirá para analisar a Presença Social do cursista e fornecer informações para a avaliação.

O artigo está organizado com a seguinte estrutura: a seção seguinte apresenta conceitos de Presença Social (PS) na EaD e sua importância para os avaliadores. É apresentado ainda dentro desta seção, o modelo de avaliação Learning Vectors (LV). A seção 3 trata de lógica difusa, seus conceitos e aplicações. A seção 4 mostra e discute os procedimentos metodológicos. Na seção 5 faz-se a análise de resultados e, por fim, relatam-se as conclusões do estudo.

\section{Presencialidade Virtual ou Presença Social}

Para Bizzo (2009), a presencialidade remete a ideia de conectado, sincronizado. Assim a comunicação em tempo real entre um professor e um aluno, seja em curta ou longa distância, é considerada como presencial.

Segundo Valente (2000), aprendizagem e educação são processos "presenciais", ou seja, exigem o encontro, a troca, a cooperação que podem ocorrer mesmo com os sujeitos estando "a distância". Nesse sentido, o termo presencialidade pode significar também, o "estar juntos virtualmente" [Valente 2000].

Diante desse cenário o espaço físico da sala de aula está dando lugar ao ciberespaço, ou melhor, a construção de "redes de aprendizagem", onde professores e alunos aprendem, interagem e cooperam entre si [Lévy and Guimarães 1998].

Segundo Vidal and Maia (2015), para integrar-se ao ciberespaço é necessário a utilização dos múltiplos mecanismos de comunicação no campo educacional através da internet (e-mail, chats, news, web conferências e fóruns) que ampliam as possibilidades da aprendizagem dinâmica e participativa, transpondo o conceito tradicional de tempo e espaço e "estabelecendo novas pontes entre o estar juntos fisicamente e virtualmente" [Moran 2008 aput Sales 2010].

A presencialidade virtual na EaD é vital para o desenvolvimento e conclusão de um curso. Por se tratar de um ambiente fora da sala de aula, fora do contexto tradicional de aprendizagem aluno/professor, é necessário um contato através do AVA, e isso se dá por meio da tecnologia, onde, mesmo estando longe e separados fisicamente, estão bem próximos virtualmente, tirando dúvidas, passando conteúdos, interagindo, etc. Essa proximidade virtual equivale a uma distância psicológica ao que Moore (1993) denomina de "distância transacional".

A pesquisa de presencialidade online ou Presença Social surgiu a partir de um estudo mediado human-to-human na pesquisa de Short et al. [apud Kim 2011]. Short e colegas definem a Presença Social como "o grau de relevância da outra pessoa na interação". Ou seja, a definição enfoca a consciência dos usuários de tecnologia da outra pessoa em um ambiente mediado.

À medida que as tecnologias evoluem, a noção de presencialidade social aumenta [Kim 2011], assim, as pessoas experimentariam a Presença Social em qualquer tipo de 
VI Congresso Brasileiro de Informática na Educação (CBIE 2017)

Anais do XXVIII Simpósio Brasileiro de Informática na Educação (SBIE 2017)

ambiente virtual e em qualquer tipo de contexto de interação mediado pela tecnologia. Presença Social também envolve afetividade, conceituado por Bastos et al. (2011), como a manifestação verbal e percepção da afetividade e interatividade dos sujeitos em relação ao grupo. Essa manifestação e percepção de PS acontecem, sobretudo, nas trocas discursivas em e-mails, fóruns e chats. São nesses espaços de relacionamentos que o sentimento de pertença e a afetividade dos sujeitos se mostram mais explícitos [Bastos et al. 2011]

A seguir, trata-se de um modelo de avaliação que será a base dessa pesquisa no intuito de aferir a Presença Social.

\subsection{O Modelo LV}

O Modelo Learning Vectors (Modelo LV) foi pensado como um modelo de função dupla de suporte e de gerenciamento para ser aplicado a AVA e, dessa forma, permitir um acompanhamento qualitativo e quantitativo do desempenho/prática dos estudantes de EaD [Sales 2010].

O Modelo LV aplica-se ao suporte e gerenciamento da avaliação formativa em AVA. "Eles podem possibilitar feedbacks constantes das ações do aprendiz no ambiente virtual de aprendizagem, além de reduzir a sobrecarga gerada para o professor-tutor em cursos a distância" [Sales et al. 2008]. Está fundamentado na interação e aplicação de vetores e equações trigonométricas em sua especificação, e fundamentam-se no modelo não linear e psicomatemático Meta Learning [Losada 1999].

Tal modelo gerencia as notas das atividades presenciais e à distância, assim como a frequência do cursista, ainda facilita a mediação e interação Tutor - Cursista, por empregar linguagem iconográfica associada a menções qualitativas na avaliação (Figura 1). A escala utilizada para fazer menções qualitativas é construída sobre seis itens de apreciação: Muito Bom, Bom, Regular, Fraco, Não Satisfatório e Neutro, associadas a ícones (LV Ícones).

\begin{tabular}{|c|c|c|c|c|c|c|}
\hline $\begin{array}{c}\text { Menções } \\
\text { Qualitativas } \\
\text { Coeficiente do } \\
\text { Passo (CP) }\end{array}$ & $\begin{array}{c}\text { Muito } \\
\text { Bom }\end{array}$ & Bom & Regular & Fraco & $\begin{array}{c}\text { Não } \\
\text { Satisfatório }\end{array}$ & Neutro \\
\hline LV Ícones & $\ddots$ & 3 & 2 & 1 & 0 & - \\
\hline
\end{tabular}

Figura 1. Escala de Menções qualitativas e correspondência com o CP.

Sales (2010), afirma que estes "itens de apreciação da Escala de Menções relacionam-se ao nível da interação do aluno (passivo/ativo) e a sua profundidade de reflexão (domínio de conteúdos) exprimindo a subjetividade da avaliação”.

Para apresentação das notas usa representações geométricas vetoriais, denominadas de Vetores-Aprendizagem (LV), que possuem módulo fixo de 10 unidades com direção inicial $\theta=-90$ (nota mínima) até $\theta=0$ (nota máxima) e $I$ representa a variação angular total conforme (Figura 2).

O módulo do componente horizontal do $\operatorname{LV}_{x}=10 * \operatorname{Cos}[(-90+I)]$ representa às ações do aluno e sua positividade de desempenho, o componente vertical $L V_{y}=10 * \operatorname{Sen}[(-90+I)]$ e relaciona-se à negatividade de seu desempenho, esses 


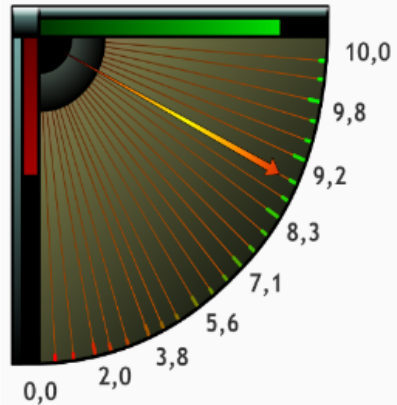

( a )

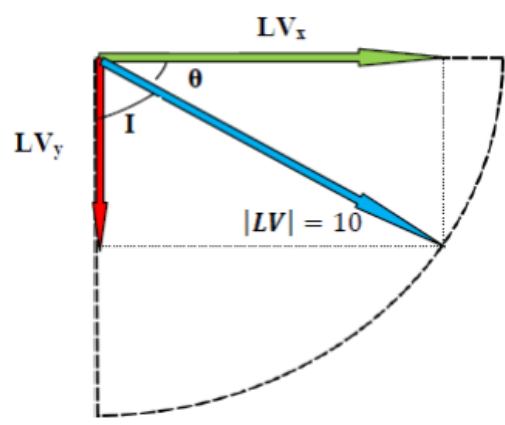

(b)

Figura 2. (a) Vetor-Aprendizagem e suas projeções (b) Módulos positividade e negatividade.

componentes refletem diretamente no rendimento e no resultado final da nota do aluno. A razão entre os fatores positividade/negatividade, contribui para a métrica pedagógica não-linear denominada Fator $\beta$, que se relaciona à natureza qualitativa desse modelo de avaliação [Sales et al. 2012].

A Seção a seguir, trata da modelagem do LV a partir da lógica difusa no intuito de capturar melhor as subjetividades presentes no ato de avaliar a Presença Social.

\section{Lógica Difusa ou Nebulosa}

A Lógica Fuzzy tem como principal objetivo quantificar valores imprecisos, ambíguos e vagos do cotidiano humano. Expressões do tipo (alto, médio, baixo e pouco ) apresentam uma certa subjetividade, sendo difícil de representar corretamente. Por exemplo, o termo (um minuto por favor) é referente a um tempo baixo, porém representar valores diferente para pessoas diferentes.

Ao contrário da Lógica convencional, a Lógica Fuzzy utiliza a ideia de que todas as coisas admitem (temperatura, altura, velocidade, etc.) graus de pertinências. Com isso, a Lógica Fuzzy tenta modelar o senso de palavras, tomada de decisão ou senso comum do ser humano [Marro et al. 2010]. A relação de pertinência é um conceito muito importante na "Teoria dos Conjuntos", a pertinência de um dado elemento com relação a um conjunto refere-se ao fato de tal elemento pertencer ou não a esse conjunto [Marro et al. 2010]. De forma ilustrativa, na Figura 3(a), representa-se um exemplo típico da teoria clássica e descreve-se o tempo de participação em um fórum de uma pessoa através de três conjuntos: baixo, médio e alto.

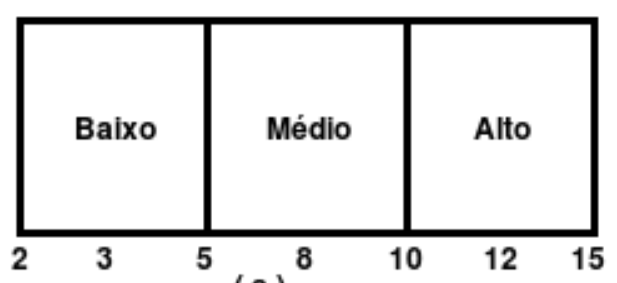

(a)

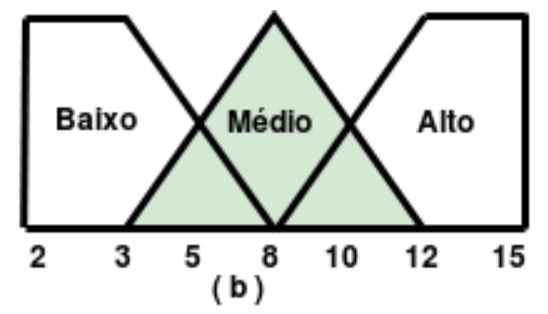

(b)

Figura 3. Representação no tempo em minutos de uma pessoa, sob o ponto de vista da Lógica convencional (a) e da Lógica Fuzzy (b). 
Nesse exemplo, dado um elemento $x$ qualquer, o mesmo pertencerá a um dos conjuntos do gráfico; por exemplo, se $x=3$ min, então $x$ pertence ao conjunto baixo e não aos demais, ou seja, um elemento pertence ou não a um determinado conjunto e, além disso, tal elemento não pertence a mais de um conjunto.

Ainda tomando como exemplo o gráfico da Figura 3(b), dados dois elementos $x_{1}=$ 9 min. e $55 \mathrm{seg}$. e $x_{2}=10 \mathrm{~min}$. e $5 \mathrm{seg}$. se a lógica clássica for utilizada, esses dois elementos pertencem a classes diferentes, $x_{1}$ pertencendo à classe médio e $x_{2}$ à classe alta. No entanto, na realidade fica difícil de dizer que duas pessoas com uma diferença tão pequena de tempo pertençam a classes diferentes.

Por outro lado, na Lógica Fuzzy, tanto o $x_{1}$ quanto o $x_{2}$ tem graus de pertinências aos conjuntos fuzzy definidos, que podem variar de 0 a 1. Zadeh (1965) definiu os conjuntos difusos mais amplamente, como a existência de elementos que pertencem a um conjunto mais do que outros.

A mente humana opera com conceitos subjetivos, tais como alto, baixo, velho e novo, que são incorporados em classes de objetos na teoria fuzzy, onde a pertinência ou não de um elemento a um conjunto, dá-se de forma gradual e não abrupta [Zadeh 1965].

Os conjuntos difusos são úteis para representar conceitos com limites imprecisos. Esta capacidade de representar conceitos imprecisos naturalmente é importante para sistemas de avaliação, pois nos permite representar tipos de conceitos usados pelos humanos, como baixo, médio, alto, jovem, pequeno, gordo, etc. [Yager and Filev 1994]. Se nosso universo de discurso for o conjunto de notas de um aluno, conceitos como: muito bom, bom, regular e fraco são representados de uma forma mais natural para o conjunto difuso.

\section{Procedimento Metodológico}

Para a realização desta pesquisa foi utilizada a base de dados de alguns fóruns de discussões da disciplina de Avaliação em Educação a Distância do curso de Especialização em EaD, da Universidade Federal do Ceará - UFC, de onde se extraiu informações de acesso de 36 alunos para montar a base de inferência Fuzzy.

Foi utilizado a toolbox do MatLab ${ }^{\circledR}$, denominada Logic Toolbox, para encontrar o fator presencialidade de cada aluno no Fórum LV.

O modelo proposto por Arias et al. (2012), o qual utiliza-se apenas de uma máquina fuzzy, foi tomado como referência para realização do estudo. Foram utilizados os seguintes universos do discurso: Tempo de permanência no fórum, quantidade de interações, nota final e quantidade de citações ou referências do aluno no fórum por outros participantes.

Todas as informações extraídas do Fórum LV contabilizaram 437 interações entre os 36 alunos no período de uma semana de participação.

Foram escolhidos 13 alunos que pudessem representar as quatro classes (muito bom, bom, regular e fraco). Esse conjunto representa bem o universo dos alunos para todas as faixas de notas. Os valores obtidos foram normalizados para o intervalo entre 0 a 1, com o objetivo de simplificar a inferência fuzzy, conforme Tabela 1. 
VI Congresso Brasileiro de Informática na Educação (CBIE 2017)

Anais do XXVIII Simpósio Brasileiro de Informática na Educação (SBIE 2017)

Tabela 1. Base de Informações dos alunos

\begin{tabular}{|l|l|c|c|c|c|c|c|c|c|c|c|c|c|}
\hline \multicolumn{10}{|c|}{ Base de informações dos alunos } \\
\hline Ident. do aluno & A1 & A2 & A3 & A4 & A5 & A6 & A7 & A8 & A9 & A10 & A11 & A12 & A13 \\
\hline Tempo & 0.75 & 1 & 0.63 & 0.58 & 0.49 & 0.36 & 0.71 & 0.89 & 0.53 & 0.61 & 0.29 & 0.45 & 0.11 \\
\hline Qtd referências & 0.28 & 0.34 & 0.52 & 0.52 & 1 & 0.07 & 0.07 & 0.34 & 0.31 & 0.21 & 0.07 & 0.17 & 0.17 \\
\hline Qtd interações & 0.78 & 0.67 & 1 & 0.22 & 0.22 & 0.33 & 0.22 & 0.22 & 0.44 & 0.33 & 0.11 & 0.11 & 0.11 \\
\hline Nota geral & 10 & 10 & 10 & 9.2 & 9.2 & 9.7 & 9.2 & 9.8 & 9.7 & 9.7 & 7.1 & 5.6 & 5.6 \\
\hline
\end{tabular}

Foram coletadas informações de seus tempos de acesso ao fórum, quantidade de interações no fórum, quantidade de citações/referências pelos colegas e nota de rendimento na escala LV. Com base nessas informações, foi montada uma planilha onde três especialistas em Educação a Distância atribuíram uma nota de presencialidade para cada aluno. Os valores extraídos da base de dados foram convertidos para valores dentro do intervalo de 0 a 1 para simplificar a inferência fuzzy.

Para tal fim, foi criado um Fuzzy Logic Controller (FLC), Figura 4, onde foram definidos os conjuntos difusos para cada entrada (tempo, quantidade de interações, quantidade de referências e notas), a máquina de inferência fuzzy, e a saída, representada pelo fator de presencialidade definido conforme representação do Modelo LV.
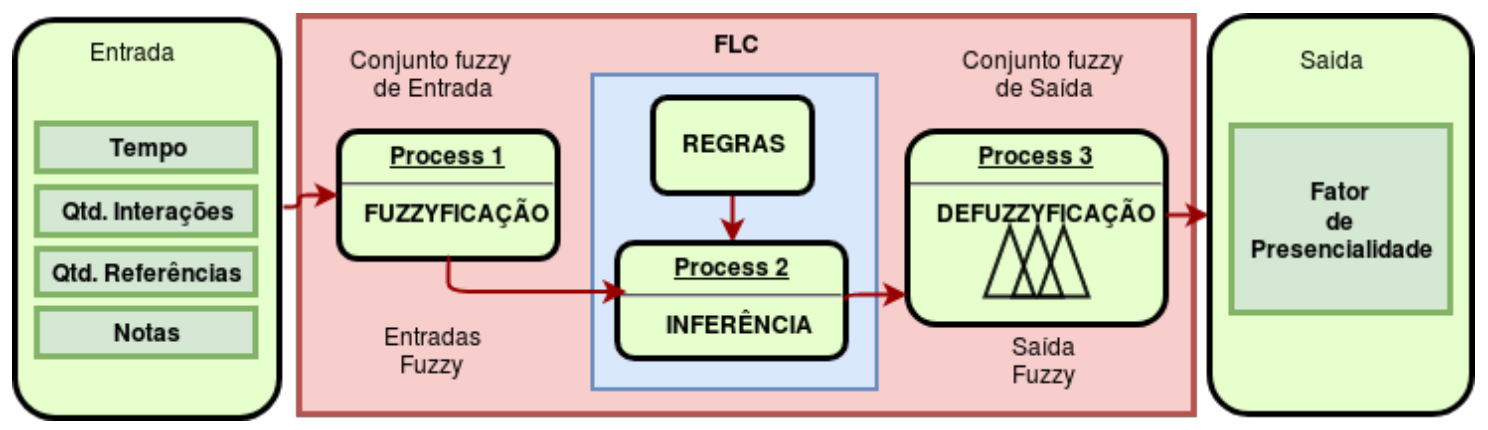

Figura 4. Fuzzy Logic Control - Representa as entradas, processo de inferência e saída.

O processo de inferência difusa envolve a utilização de funções de pertinência, operações lógicas ( $A N D$ e OR) e estruturas de decisão (IF-THEN). Com base nas informações dos especialistas, foram criadas 276 regras que fazem parte do motor de inferência difuso, para os quatro fatores de avaliação: Tempo (T), quantidade de citações pelos colegas $(\mathrm{QR})$, quantidade de interações $(\mathrm{QI})$ e nota do fórum $(\mathrm{N})$.

Tabela 2. Conjunto de Regras de Inferências

\begin{tabular}{|c|c|c|c|c|c|c|c|c|c|c|}
\hline Regras & \multirow{5}{*}{ If } & Ent. 1 & \multirow{5}{*}{ and } & Ent. 2 & \multirow{5}{*}{ and } & Ent. 3 & \multirow{5}{*}{ and } & Ent. 4 & \multirow{5}{*}{ Then } & Saída \\
\hline 1 & & $\mathrm{~T}=$ baixo & & $\mathrm{QR}=$ baixo & & QI=baixo & & $\mathrm{N}=$ baixo & & Baixo \\
\hline 2 & & $\mathrm{~T}=$ baixo & & $\mathrm{QR}=$ baixo & & QI=baixo & & $\mathrm{N}=$ baixo & & Baixo \\
\hline$\ldots$ & & & & & & & & & & \\
\hline 276 & & T=M.bom & & QR=M.bom & & QI=M.bom & & $\mathrm{N}=$ alta & & Ótimo \\
\hline
\end{tabular}

As regras difusas são a representação do conhecimento do professor especialista e que servirá para determinar as saídas nos sistema Fuzzy. 
VI Congresso Brasileiro de Informática na Educação (CBIE 2017)

Anais do XXVIII Simpósio Brasileiro de Informática na Educação (SBIE 2017)

A Figura 5 apresenta o editor de funções de pertinência imerso dentro de cada FLC. Neste editor foram criados os conjuntos difusos que irão representar as variáveis de entrada.

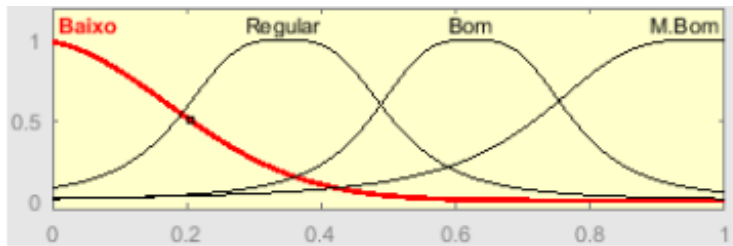

(a)

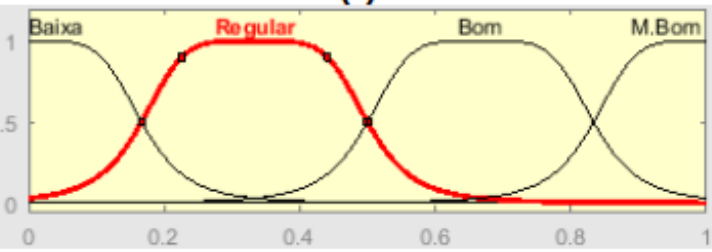

(c)

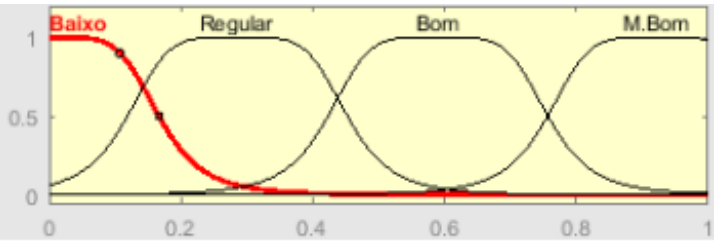

(b)

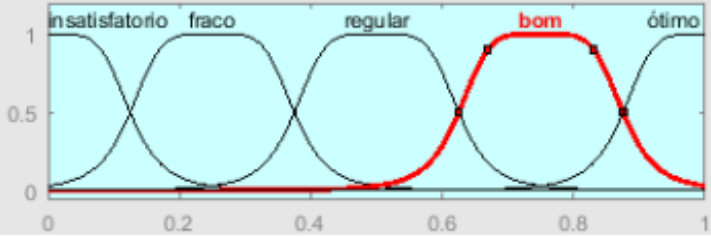

(d)

Figura 5. Representação das entradas e saída do FLC. T (a), QR (b), QI (c) e saída do sistema (d).

\section{Análise e Discussão dos Resultados}

O sistema difuso é alimentado com os valores de entrada da tabela 1, as regras são ajustadas para a máquina de inferência e são obtidos os resultados da avaliação da presencialidade. Serão apresentados os dados do sistema difuso e uma comparação com a nota final do aluno.

Foi possível obter com maior exatidão o desempenho de cada aluno levando em consideração o tempo de participação, a quantidade de interações, a quantidade de referências e a nota obtida. Na Tabela 3 apresentam-se os resultados finais do processo de avaliação difusa de cada aluno, e também a nota da atividade.

Tabela 3. Notas do Fórum e Fator Presencialidade

\begin{tabular}{|l|l|l|l|l|l|l|l|l|l|l|l|l|l|}
\hline \multicolumn{10}{|c|}{ Notas e Fator Presencialidade } \\
\hline Id. do aluno & A1 & A2 & A3 & A4 & A5 & A6 & A7 & A8 & A9 & A10 & A11 & A12 & A13 \\
\hline Nota do Fórum & 10 & 10 & 10 & 9.2 & 9.2 & 9.7 & 9.2 & 9.8 & 9.7 & 9.7 & 7.1 & 5.6 & 5.6 \\
\hline Presencialidade & 7.86 & 9.42 & 9.93 & 8.82 & 9.37 & 7.97 & 8.58 & 8.82 & 8.52 & 8.83 & 4.21 & 5.68 & 4.17 \\
\hline
\end{tabular}

No Tabela 3, observa-se que o aluno de identificação A13 obteve nota do fórum 5.6 e não participou ativamente do fórum, fazendo apenas uma postagem (interação) que não foi citada e referenciada pelos colegas conforme Tabela 1 . Na avaliação da presencialidade, seu rendimento ainda é menor, obtendo uma pontuação de 4.17, pois os fatores participação e interação praticamente foram inexistentes. Os alunos A1, A2 e A3 participaram ativamente do fórum e receberam em suas avaliações a nota máxima (10). Já na avaliação da presencialidade, foi apresentada uma variação para menor do aluno A1 e uma pequena variação entre os alunos A2 e A3. O Aluno A3 manteve praticamente o mesmo desempenho. Apesar de as notas finais terem sido iguais, o fator presencialidade foi bem diferente. Isso ocorreu principalmente pela quantidade de interações. $\mathrm{O}$ aluno 
VI Congresso Brasileiro de Informática na Educação (CBIE 2017)

Anais do XXVIII Simpósio Brasileiro de Informática na Educação (SBIE 2017)

A6 obteve como nota de participação do fórum, o valor de 9,7, nota muito próxima dos alunos A1, A2 e A3, porém ao verificar o fator presencialidade, seu rendimento foi de 7,97 uma queda de quase 2 pontos, representando uma redução de $20 \%$ da nota. Essa redução foi fruto da baixa visibilidade das postagens pelos outros alunos, pois foi pouco citado e referenciado. Isso pode significar que a informação postada no fórum foi de baixa relevância para a discussão. Finalmente o aluno A5 que obteve como nota o valor de 9,2 e seu fator presencialidade foi de 9,37 tendo um crescimento pequeno, mas muito significativo. O aluno teve poucas interações, e consequentemente seu tempo foi baixo, mas o impacto dessas interações no grupo proporcionou muitas referências e discussões, pois muitos alunos faziam uso da referida postagem em suas contribuições.

No Figura 6, apresenta-se o gráfico de desempenho da nota do fórum e do fator presencialidade de toda a turma. É possível fazer também um comparativo entre a amostra que serviu para o treinamento da máquina e a da validação.

Desempenho por Nota e Presencialidade

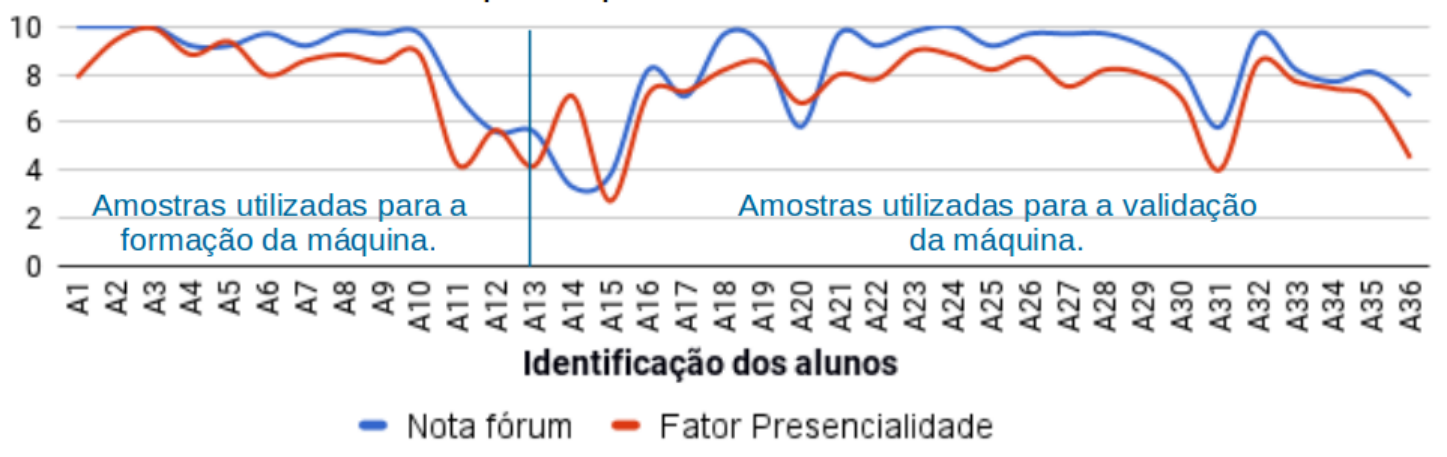

Figura 6. Gráfico de comparação Nota do fórum e Fator Presencialidade

\section{Conclusão}

Como indicado neste estudo, buscou-se contemplar os modelos utilizados da lógica nebulosa para aplicação na avaliação da presencialidade de alunos em um Fórum LV. O uso da lógica difusa nos permite dar uma maior flexibilidade no momento de determinar essa presencialidade, já que é possível mensurar a participação dos mesmos nos AVA.

Neste estudo, foram utilizados critérios como tempo, quantidade de interações, nota e quantidade de citações pelos colegas para verificação das interações discursivas feitas no Fórum LV dos ambientes de aprendizagem online. Foi possível conceber um modelo de avaliação da presencialidade que possa realizar uma classificação mais justa dos alunos em $\mathrm{EaD}$, levando em conta os fatores apresentados.

Considerando o exposto apresentado neste trabalho, pretende-se aprimorar a metodologia com a utilização de outros fatores referentes à presencialidade social (afetividade, expressões não verbais e o "internetês"). E como trabalhos futuros, propõe aplicar a modelagem ora proposta em outras ferramentas, como Chats, Wiki do AVA Moodle.

\section{Agradecimentos}

Este pesquisa é apoiada pelo PPGCC/IFCE e pela Fundação Cearense de Apoio ao Desenvolvimento Científico e Tecnológico - FUNCAP (Edital 005/2014). 
VI Congresso Brasileiro de Informática na Educação (CBIE 2017)

Anais do XXVIII Simpósio Brasileiro de Informática na Educação (SBIE 2017)

\section{Referências}

Arias, R. A., Panhan, A. M., Breda, G. D., Zarpelão, B. B., and Mendes, L. d. S. (2012). Avaliação multidimensional baseada em lógica difusa para ambientes de ensino mediado por computador. Revista Brasileira de Informática na Educação, 20(3):74-84.

Bai, S.-M. and Chen, S.-M. (2008). Evaluating students' learning achievement using fuzzy membership functions and fuzzy rules. Expert Systems with Applications, 34(1):399-410.

Bastos, H. P. P., Bercht, M., and Wives, L. K. (2011). Presença social e pertencimento em fóruns educacionais: manifestação e percepção de afetividade. In Brazilian Symposium on Computers in Education (Simpósio Brasileiro de Informática na Educação-SBIE).

Bizzo, N. (2009). Ensino de ciências e ead. IN:LITTO, F.; FORMIGA, M. (Orgs.). Educação a distância: o estado da arte. São Paulo: Pearson Education do Brasil.

Kim, J. (2011). Developing an instrument to measure social presence in distance higher education. British Journal of Educational Technology, 42(5):763-777.

Kim, J., Song, H., and Luo, W. (2016). Broadening the understanding of social presence: Implications and contributions to the mediated communication and online education. Computers in Human Behavior, 65:672-679.

Lévy, P. and Guimarães, M. M. A. (1998). A máquina universo: criação, cognição e cultura informática.

Losada, M. (1999). The complex dynamics of high performance teams. Mathematical and computer modelling, 30(9-10):179-192.

Marro, A. A., Souza, A. d. C., Cavalcante, E. d. S., Bezerra, G. S., and NUNES, R. (2010). Lógica fuzzy: conceitos e aplicações. Natal: Universidade Federal do Rio Grande do Norte (UFRN).

Moore, M. G. (1993). Theory of transactional distance. Theoretical principles of distance education, 1:22-38.

Saleh, I. and Kim, S.-i. (2009). A fuzzy system for evaluating students' learning achievement. Expert systems with Applications, 36(3):6236-6243.

Sales, G. L. (2010). Learning vectors (lv): um modelo de avaliação da aprendizagem em ead online aplicando métricas não-lineares. Tese de doutorado, Universidade Federal do Ceará-UFC.

Sales, G. L., Barroso, G. C., and Soares, J. M. (2008). O indicador de aprendizagem learning vectors como instrumento automatizado de avaliaçao para suporte a aprendizagem em ead. In Anais do Workshop de Informática na Escola, volume 1.

Sales, G. L., Barroso, G. C., and Soares, J. M. (2012). Learning vectors (lv): Um modelo de avaliação processual com mensuração não-linear da aprendizagem em ead online. Revista Brasileira de Informática na Educação, 20(1):60.

Silva, J. K. K. d. (2012). Automatização do processo de identificação de presença social em fóruns e chats. In Brazilian Symposium on Computers in Education (Simpósio Brasileiro de Informática na Educação-SBIE).

Valente, J. A. (2000). Educação a distância: uma oportunidade para mudança no ensino. Educação a distância no Brasil na era da Internet. São Paulo: Anhembi Morumbi Editora, pages 97-122.

Vidal, E. M. and Maia, J. E. B. (2015). Introdução à educação a distância. - 2. ed. rev. Fortaleza : EdUECE.

Yager, R. R. and Filev, D. P. (1994). Essentials of fuzzy modeling and control. New York. Zadeh, L. A. (1965). Fuzzy sets. Information and control, 8(3):338-353. 